\title{
Relaxation oscillation in one-parameter families of Liénard equations
}

\section{Pedro Toniol Cardin ${ }^{1}$}

Faculdade de Engenharia de Ilha Solteira, Universidade Estadual Paulista (UNESP)

Douglas Duarte Novaes ${ }^{2}$

Instituto de Matemática, Estatística e Computação Científica, Universidade Estadual de Campinas (UNICAMP)

\begin{abstract}
In this work we study the asymptotic behavior of periodic solutions in oneparameter $(\lambda>0)$ families of Liénard differential equations, for large values of $\lambda$. Under certain conditions, we prove the existence of a relaxation oscillation, occurring upon variation of the parameter.
\end{abstract}

Keywords. Liénard equation, periodic solution, relaxation oscillation.

\section{Introduction and main result}

Relaxation oscillation is a kind of global phenomenon that typically occurs in slow-fast differential systems (see $[4,6,7]$ ). It is characterized by a special type of periodic orbit consisting of long periods of quasi-static motion interspersed with short periods of rapid transition. A prototypical model where relaxation oscillation occurs is the classical van der Pol equation which models the oscillations of a triode vacuum tube (see $[5,8]$ ).

In this work we consider one-parameter $\lambda>0$ families of Liénard differential equations of the form

$$
x^{\prime \prime}+\lambda f(x) x^{\prime}+x=0 .
$$

In (1) the prime indicates derivative with respect to the time $t$. Note that for $f(x)=x^{2}-1$ we get the van der Pol equation.

Taking $x^{\prime}=y$ the equation (1) can be converted into the first order differential system

$$
x^{\prime}=y, \quad y^{\prime}=-x-\lambda f(x) y .
$$

In this work we are concerned with the study on the existence and uniqueness of limit cycles of system (2) for all positive values of $\lambda$, and mainly on the asymptotic behavior of such limit cycle for large values of $\lambda>0$.

\footnotetext{
${ }^{1}$ pedro.cardin@unesp.br.

2 ddnovaes@ime.unicamp.br.
} 
The assumptions we are going to make are as follows. Consider the auxiliary function

$$
F(x)=\int_{0}^{x} f(s) d s
$$

and assume the hypotheses:

(H1) $f$ is a $C^{2}$-function on $\mathbb{R}$ having precisely two zeros, $x_{M}$ and $x_{m}$, such that $x_{M}<$ $0<x_{m}$ and $f^{\prime}\left(x_{M}\right)<0<f^{\prime}\left(x_{m}\right)$.

(H2) The straight lines $y=F\left(x_{M}\right)$ and $y=F\left(x_{m}\right)$, passing through the points $A=$ $\left(x_{M}, F\left(x_{M}\right)\right)$ and $B=\left(x_{m}, F\left(x_{m}\right)\right)$, intersect the graphic of $F$ at the points $A^{\prime} \neq A$ and $B^{\prime} \neq B$, respectively.

(H3) The differential system (2) has at most one limit cycle.

Remark 1.1. Hypothesis (H3) is a qualitative assumption on the system (2). It is worthwhile to say that there are many analytical conditions for which it holds (see, e.g., the books $[9,10])$.

In what follows we define some preliminary objects before stating our main result. Take $x_{1}<0<x_{2}$ such that $A^{\prime}=\left(x_{2}, F\left(x_{M}\right)\right)$ and $B^{\prime}=\left(x_{1}, F\left(x_{m}\right)\right)$. From (H2) it follows that $x_{1} \neq x_{m}$ and $x_{2} \neq x_{M}$. From (H1) and (H2) we find exactly two zeros of $F$, $x_{1}^{*}$ and $x_{2}^{*}$, such that $x_{1}^{*}<0<x_{2}^{*}$. Clearly $x_{1}<x_{1}^{*}<x_{M}$ and $x_{m}<x_{2}^{*}<x_{2}$. Let $\overline{A A^{\prime}}$ and $\overline{B B^{\prime}}$ be the line segments joining $A$ to $A^{\prime}$ and $B$ to $B^{\prime}$, respectively. Let $\widetilde{A^{\prime} B}$ and $\widetilde{B^{\prime} A}$ be the pieces of $\operatorname{Gr}(F)$ joining $A^{\prime}$ to $B$ and $B^{\prime}$ to $A$, respectively. We define $\Gamma_{0} \subset \mathbb{R}^{2}$ as being the closed curve given by the union $\Gamma_{0}=\overline{A A^{\prime}} \cup \widetilde{A^{\prime} B} \cup \overline{B B^{\prime}} \cup \widetilde{B^{\prime} A}$, see Figure 1 .

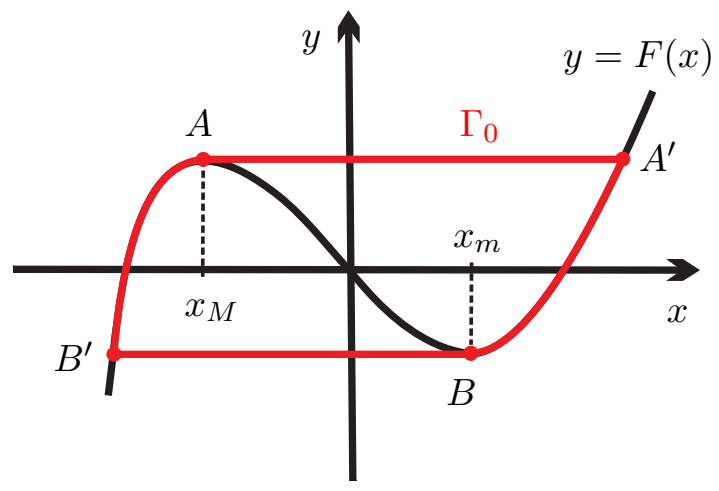

Figura 1: The closed curve $\Gamma_{0}$.

Our main result is the following one.

Theorem 1.1. Under the hypotheses (H1), (H2), and (H3), the following statements hold:

(i) For every $\lambda>0$, the differential system (2) has a unique stable limit cycle $\Phi(\lambda)$ depending continuously on $\lambda$. 
(ii) Let $P_{\lambda}(x, y)=(x, F(x)+y / \lambda)$ be an one-parameter family of diffeomorphims. Then

$$
\lim _{\lambda \rightarrow \infty} d_{H}\left(\Phi(\lambda), P_{\lambda}^{-1}\left(\Gamma_{0}\right)\right)=0 .
$$

where $d_{H}(\cdot, \cdot)$ denotes the Hausdorff distance.

Remark 1.2. The limit given in (4) means that, for sufficiently large values of $\lambda$, after a change of coordinates, the limit cycle $\Phi(\lambda)$ approaches the closed curve $\Gamma_{0}$.

\section{Preliminary}

In what follows we describe some ideas involving slow-fast singularly perturbed systems and the relaxation oscillation theory occurring in such systems. Consider a slow-fast differential system in $\mathbb{R}^{2}$ of the form

$$
\mu \frac{d x}{d s}=g(x, y, \mu), \quad \frac{d y}{d s}=h(x, y, \mu),
$$

where $g$ and $h$ are $C^{r}$-functions with $r \geq 3$. For $\mu>0$, system (5) is equivalent to

$$
\frac{d x}{d \tau}=g(x, y, \mu), \quad \frac{d y}{d \tau}=\mu h(x, y, \mu),
$$

which is obtained after the time rescaling $\tau=s / \mu$. System (5) is the slow system and (6) is the fast system. A usual way to treat with slow-fast systems is through the geometric singular perturbation theory (GSPT), see $[1,3]$. The idea is to study the (limiting) slow and fast dynamics separately and then combine results on these two limiting behaviours in order to obtain information on the dynamics of the full system (5) (or (6)) for small values of $\mu$.

Letting $\mu \rightarrow 0$ in (5) and in (6) we obtain, respectively, the reduced problem

$$
0=g(x, y, 0), \quad \frac{d y}{d s}=h(x, y, 0)
$$

and the layer problem

$$
\frac{d x}{d \tau}=g(x, y, 0), \quad \frac{d y}{d \tau}=0 .
$$

The phase space of (7) is the so-called critical manifold defined by $\mathcal{S}=\left\{(x, y) \in \mathbb{R}^{2}\right.$ : $g(x, y, 0)=0\}$. On the other hand, $\mathcal{S}$ is the set of equilibria for the layer problem (8). Among other things, Fenichel theory [3] (see also [1]) guarantees the persistence of a normally hyperbolic subset $\mathcal{S}_{0} \subseteq \mathcal{S}$ as a slow manifold $\mathcal{S}_{\mu}$ of (5) (or (6)) for small enough values of $\mu>0$. Moreover, the flow on $\mathcal{S}_{\mu}$ is a small perturbation of the flow of $(7)$ on $\mathcal{S}_{0}$. Normal hyperbolicity of $\mathcal{S}_{0}$ means that $(\partial g / \partial x)(x, y) \neq 0$ for all $(x, y) \in \mathcal{S}_{0}$. That is, $\mathcal{S}_{0}$ is normally hyperbolic if for each $(\bar{x}, \bar{y}) \in \mathcal{S}_{0}$, we have that $\bar{x}$ is a hyperbolic equilibrium point of $(d x / d \tau)=g(x, \bar{y}, 0)$.

Generically, a non-normally hyperbolic point is a fold point of $\mathcal{S}$. In the case when the critical manifold $\mathcal{S}$ has non-normally hyperbolic points, interesting global phenomena can 

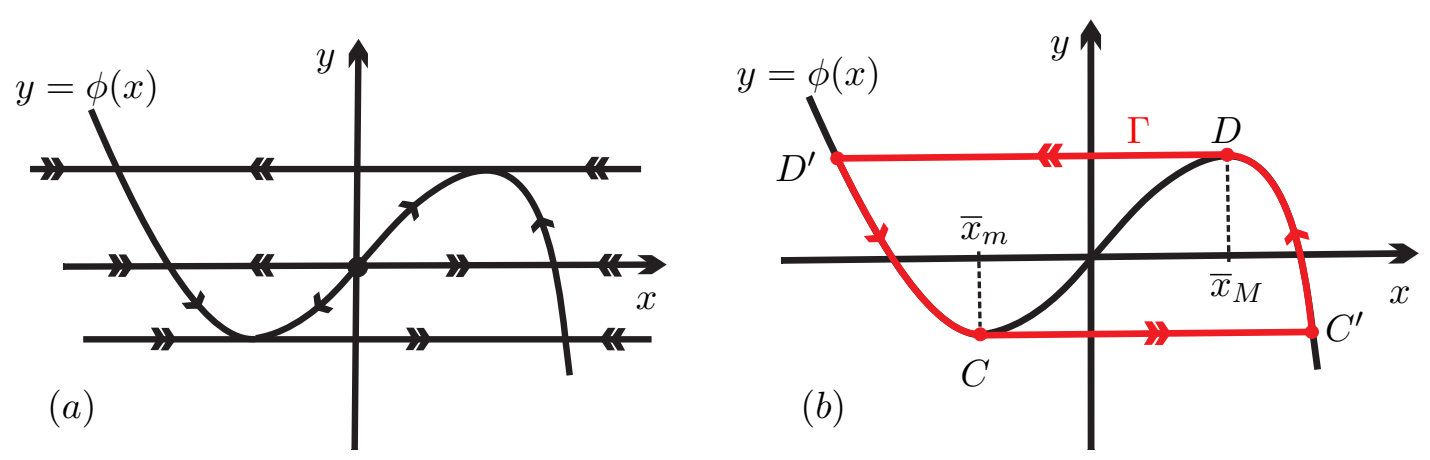

Figura 2: Panel (a) illustrates the phase portraits of (7) and (8) (double and single arrows indicate the direction of the fast and slow flows, respectively), under the hypotheses (C1)-(C4). Panel (b) illustrates the singular trajectory $\Gamma$.

occur. For instance, an interesting kind of global phenomenon is the relaxation oscillations. A relaxation oscillation is a periodic solution $\Gamma_{\mu}$ of the slow-fast system (5) that converges to a singular trajectory $\Gamma_{0}$, when $\mu \rightarrow 0$, with respect to Hausdorff distance. A singular trajectory means a curve obtained as concatenations of trajectories of the reduced and layer problems (with a consistent orientation) forming a closed curve.

In what follows we describe a well known prototypical situation where a relaxation oscillation exists (see, e.g., $[4,6,7]$ ). The following assumptions will be considered.

(C1) $\mathcal{S}$ can be written in the form $y=\phi(x)$ and the function $\phi$ has precisely two critical points, one minimum $\bar{x}_{m}$ and one maximum $\bar{x}_{M}$, both non-degenerate (folds).

(C2) The fold points are generic, i.e.

$$
\begin{array}{cc}
\frac{\partial^{2} g}{\partial x^{2}}\left(\bar{x}_{m}, \phi\left(\bar{x}_{m}\right), 0\right) \neq 0, & \frac{\partial g}{\partial y}\left(\bar{x}_{m}, \phi\left(\bar{x}_{m}\right), 0\right) \neq 0, \quad h\left(\bar{x}_{m}, \phi\left(\bar{x}_{m}\right), 0\right) \neq 0, \\
\frac{\partial^{2} g}{\partial x^{2}}\left(\bar{x}_{M}, \phi\left(\bar{x}_{M}\right), 0\right) \neq 0, & \frac{\partial g}{\partial y}\left(\bar{x}_{M}, \phi\left(\bar{x}_{M}\right), 0\right) \neq 0, \quad h\left(\bar{x}_{M}, \phi\left(\bar{x}_{M}\right), 0\right) \neq 0 .
\end{array}
$$

(C3) $(\partial g / \partial x)<0$ on $\mathcal{S}_{l}=\left\{(x, \phi(x)): x<\bar{x}_{m}\right\}$ and on $\mathcal{S}_{r}=\left\{(x, \phi(x)): x>\bar{x}_{M}\right\}$, and $(\partial g / \partial x)>0$ on $\mathcal{S}_{m}=\left\{(x, \phi(x)): \bar{x}_{m}<x<\bar{x}_{M}\right\}$. This means that for the layer problem (8) the branches $\mathcal{S}_{l}$ and $\mathcal{S}_{r}$ are attracting while $\mathcal{S}_{m}$ is repelling.

(C4) The slow (reduced) flow on $\mathcal{S}_{l}$ and $\mathcal{S}_{r}$ satisfies that $(d y / d s)<0$ and $(d y / d s)>0$, respectively.

Assuming the hypotheses (C1)-(C4), Figure 2(a) illustrates the phase portraits of the reduced and layer problems $(7)$ and (8).

Let be $C=\left(\bar{x}_{m}, \phi\left(\bar{x}_{m}\right)\right)$ and $D=\left(\bar{x}_{M}, \phi\left(\bar{x}_{M}\right)\right)$. Let $C^{\prime}$ and $D^{\prime}$ be the points of intersection of the straight lines $y=\phi\left(\bar{x}_{m}\right)$ and $y=\phi\left(\bar{x}_{M}\right)$ with $\mathcal{S}_{r}$ and $\mathcal{S}_{l}$, respectively. Consider $\Gamma$ the singular trajectory defined as the union of the fast fibers joining $C$ to $C^{\prime}$ and $D$ to $D^{\prime}$ and of the two pieces of the critical manifold $\mathcal{S}$ joining $C^{\prime}$ to $D$ and $D^{\prime}$ 
to $C$. See Figure 2(b). Let $V$ be a small tubular neighborhood of $\Gamma$. Then, under the assumptions (C1) $-(\mathbf{C 4})$, for $\mu$ small enough, system (5) admits a unique stable limit cycle $\Gamma_{\mu} \subset V$ which converges to $\Gamma$ in the Hausdorff distance as $\mu \rightarrow 0$ (see $[4,6,7]$ ).

\section{Proof of Theorem 1.1}

Proof of Theorem 1.1. To prove item (i) we will use the Dragilëv's Theorem [2] (see also Theorem 5.1 of [9]). Dragilëv's Theorem considers systems of the form

$$
x^{\prime}=y, \quad y^{\prime}=-g(x)-f(x) y,
$$

and assumes the following conditions:

(B1) The functions $F(x)$ and $g(x)$ are locally Lipschitz, where $F(x)=\int_{0}^{x} f(s) d s$.

(B2) $x g(x)>0$ for $x \neq 0, G( \pm \infty)=+\infty$, where $G(x)=\int_{0}^{x} g(s) d s$.

(B3) There exist $a_{1}<0<a_{2}$, such that $F(x)>0$, for $a_{1} \leq x<0$, and $F(x)<0$, for $0<x \leq a_{2}$.

(B4) There exist $k>\max \left\{-a_{1}, a_{2}\right\}$, and $b_{1}<b_{2}$ such that $F(x) \leq b_{1}$ if $x<-k$, and $F(x) \geq b_{2}$ if $x>k$.

Under the above conditions, Dragilëv's Theorem states that the differential system (9) has at least one stable limit cycle.

From the hypothesis (H1), $F(x)$ is a differentiable function and, for the differential system $(2), g(x)=x$. Therefore $F(x)$ and $g(x)$ are locally Lipschitz and then condition (B1) is satisfied. Condition (B2) is trivially true because $G(x)=x^{2} / 2$. Now taking $a_{1}=x_{M}$ and $a_{2}=x_{m}$ we see that hypothesis (H2) implies condition (B3). Finally condition (B4) is assured by hypotheses (H1) and (H2) if we take $k>\max \left\{-x_{1}, x_{2}\right\}$, $b_{1}=F\left(x_{m}\right)$, and $b_{2}=F\left(x_{M}\right)$. Therefore, from Dragilëv's Theorem we have assured, for each $\lambda>0$, the existence of a stable limit cycle $\Phi(\lambda)$ of the differential system (2). Clearly hypothesis (H3) implies its uniqueness for each $\lambda>0$. The continuous dependence of $\Phi(\lambda)$ on $\lambda$ follows from basic facts of the qualitative theory of ODEs.

To prove item (ii) first we transform system (2) into a slow-fast system. For that, we define a new independent variable $s$ setting $s:=t / \lambda$. This leads to system $\dot{x}=\lambda y, \dot{y}=$ $-\lambda x-\lambda^{2} f(x) y$, where the dot means derivative with respect to $s$. Then, we apply the change of coordinates $(x, u)=P_{\lambda}(x, y)=(x, F(x)+y / \lambda)$, where $F$ is given in (3), getting the system $\left(1 / \lambda^{2}\right) \dot{x}=u-F(x), \dot{u}=-x$. Finally, setting $\mu:=1 / \lambda^{2}$ and considering $\lambda$ large enough, we get the following slow-fast system with small perturbation parameter $\mu$ :

$$
\mu \dot{x}=u-F(x), \quad \dot{u}=-x .
$$

Comparing system (10) with the general form (5), we have that $g(x, u, \mu)=u-F(x)$ and $h(x, u, \mu)=-x$. The critical manifold is given by $\mathcal{S}=\left\{(x, u) \in \mathbb{R}^{2}: u=F(x)\right\}$. From the hypothesis (H1), it follows that $F$ has precisely two critical points, $x_{M}$ and 

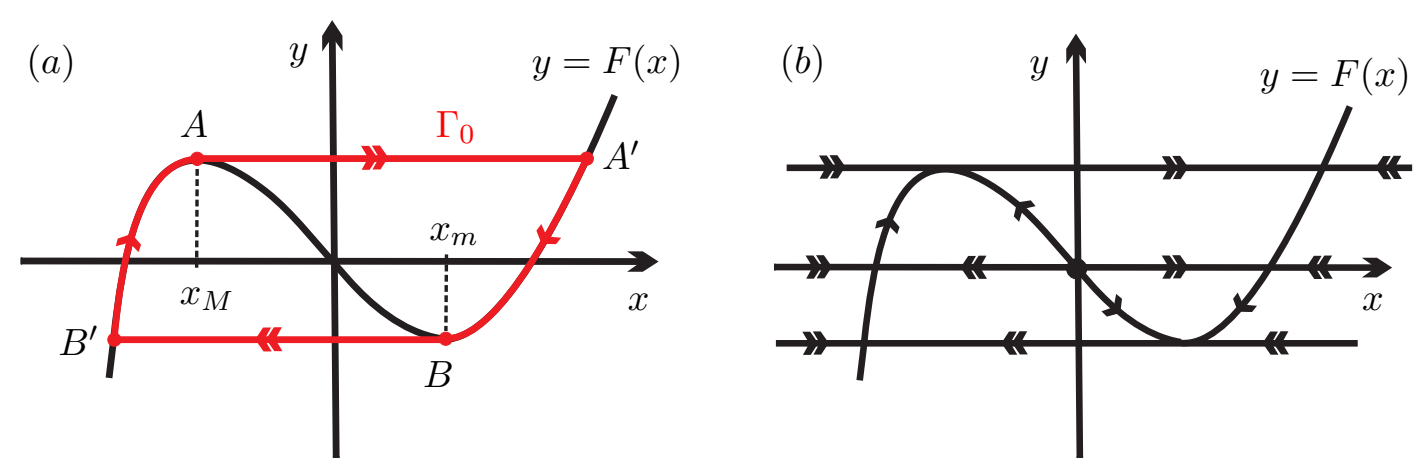

Figura 3: Panel (a) illustrates the singular trajectory $\Gamma_{0}$ and panel (b) illustrates the phase portraits of the layer and reduced problems associated with system (10). The double and single arrows indicate the direction of the fast and slow flows, respectively.

$x_{m}$, since $F^{\prime}(x)=f(x)$ for all $x \in \mathbb{R}$, and $x_{M}$ and $x_{m}$ are the only zeros of $f$. Moreover, as $F^{\prime \prime}\left(x_{M}\right)=f^{\prime}\left(x_{M}\right)<0$ and $F^{\prime \prime}\left(x_{m}\right)=f^{\prime}\left(x_{m}\right)>0$, then $A=\left(x_{M}, F\left(x_{M}\right)\right)$ and $B=$ $\left(x_{m}, F\left(x_{m}\right)\right)$ are maximum and minimum points of $F$, respectively, both non-degenerate. Also these two fold points are generic, since

$$
\begin{aligned}
& \frac{\partial^{2} g}{\partial x^{2}}(A)=-F^{\prime \prime}\left(x_{M}\right) \neq 0, \quad \frac{\partial g}{\partial u}(A)=1 \neq 0, \quad h(A)=-x_{M} \neq 0, \\
& \frac{\partial^{2} g}{\partial x^{2}}(B)=-F^{\prime \prime}\left(x_{m}\right) \neq 0, \quad \frac{\partial g}{\partial u}(B)=1 \neq 0, \quad h(B)=-x_{m} \neq 0 .
\end{aligned}
$$

From the hypothesis (H1), we can conclude that $F^{\prime}(x)<0$ for $x \in\left(x_{M}, x_{m}\right)$, and $F^{\prime}(x)>$ 0 for $x \in\left(-\infty, x_{M}\right) \cup\left(x_{m},+\infty\right)$. Therefore, for the layer problem given by $\dot{x}=u-$ $F(x), \dot{u}=0$, the branches $\mathcal{S}_{l}=\left\{(x, F(x)): x<x_{M}\right\}$ and $\mathcal{S}_{r}=\left\{(x, F(x)): x>x_{m}\right\}$ are attracting while the branch $\mathcal{S}_{m}=\left\{(x, F(x)): x_{M}<x<x_{m}\right\}$ is repelling. Relative to dynamics of the reduced problem, we have that the slow flow on $\mathcal{S}_{l}$ satisfies $\dot{u}>0$ and on $\mathcal{S}_{r}$ it satisfies that $\dot{u}<0$. On $\mathcal{S}_{m}$ the reduced problem has a repeller equilibrium at the point $(0, F(0))=(0,0)$. In short, from the hypothesis $(\mathbf{H 1})$, we have that the fast and slow dynamics are as illustrated in Figure 3(b).

We remark that the situation presented above involving system (10) is not exactly the same as in prototypical situation described from the assumptions (C1)-(C4). In this last case the critical manifold $\mathcal{S}$ is $\mathrm{S}$-shaped while in our case $\mathcal{S}$ has the shape of a $\mathrm{S}$ but reflected. Mathematically speaking, the two situations coincide after a reflection $(X, Y)=(-x, y)$ on the $y$-axis.

Let $U$ be a small tubular neighborhood of $\Gamma_{0}$. Then, under the hypotheses (H1) and (H2) it follows that, for $\mu$ small enough, system (10) has a unique stable limit cycle $\Gamma_{\mu} \subset U$ which converges to $\Gamma_{0}$ in the Hausdorff distance as $\mu \rightarrow 0$. Consequently, using the reverse change of coordinates, we can conclude that, for $\lambda>0$ sufficiently large, there exists a unique stable limit cycle $\Phi_{1}(\lambda) \subset U$ for system (2) such that

$$
\lim _{\lambda \rightarrow \infty} d\left(\Phi_{1}(\lambda), P_{\lambda}^{-1}\left(\Gamma_{0}\right)\right)=0 .
$$

Clearly, hypothesis (H3) implies that $\Phi(\lambda)=\Phi_{1}(\lambda)$, for $\lambda>0$ sufficiently large. 


\section{Conclusion}

In this work we consider one-parameter $\lambda>0$ families of Liénard differential equations (1). For each $\lambda>0$, we prove the existence of a limit cycle $\Phi(\lambda)$ as well as its continuous dependence on $\lambda$. We also provide the asymptotic behavior of such limit cycle for large values of $\lambda>0$. When $\lambda$ assumes large values, the differential system (2) can be converted into a slow-fast system which can be treated with the techniques coming from the geometric singular perturbation theory. Under the hypotheses (H1) and (H2) we establish the existence of a relaxation oscillation approaching to the singular trajectory $\Gamma_{0}$, when the parameter $\lambda$ takes large values.

\section{Referências}

[1] P. T. Cardin and M. A. Teixeira. Fenichel theory for multiple time scale singular perturbation problems. SIAM J. Appl. Dyn. Syst., 16(3):1425-1452, 2017.

[2] A. V. Dragilev. Periodic solutions of the differential equation of nonlinear oscillations. Akad. Nauk SSSR. Prikl. Mat. Meh., 16:85-88, 1952.

[3] N. Fenichel. Geometric singular perturbation theory for ordinary differential equations. J. Differential Equations, 31(1):53-98, 1979.

[4] M. Krupa and P. Szmolyan. Relaxation oscillation and canard explosion, J. Differential Equations, 174(2):312-368, 2001.

[5] N. Minorsky. Nonlinear oscillations. D. Van Nostrand Co., Inc., Princeton, N.J.Toronto-London-New York, 1962.

[6] E. F. Mishchenko and N. K. Rozov. Differential equations with small parameters and relaxation oscillations, Mathematical Concepts and Methods in Science and Engineering, volume 13, Plenum Press, New York, 1980. Translated from the Russian by F. M. C. Goodspeed.

[7] L. S. Pontryagin. Asymptotic behavior of solutions of systems of differential equations with a small parameter in the derivatives of highest order, Izv. Akad. Nauk SSSR. Ser. Mat., 21:605-626, 1957.

[8] B. van der Pol. On relaxation oscillations, Philosophical Mag., 7:978-992, 1926.

[9] Y. Q. Ye, S. L. Cai, L. S. Chen, K. C. Huang, D. J. Luo, Z. E. Ma, E. N. Wang, M. S. Wang, and X. A. Yang. Theory of limit cycles, Translations of Mathematical Monographs, volume 66, American Mathematical Society, Providence, RI, second edition, 1986.

[10] Z. F. Zhang, T. R. Ding, W. Z. Huang, and Z. X. Dong. Qualitative theory of differential equations, Translations of Mathematical Monographs, volume 101, American Mathematical Society, Providence, RI, 1992. 